\title{
Application of Activated Charcoal in the Downstream Processing of Bacterial Olefinic Poly(3-hydroxyalkanoates)
}

\author{
Bruno Wampfler, Thomas Ramsauer, Karl Kehl, Manfred Zinn, and Linda Thöny-Meyer
}

\begin{abstract}
Medium chain length poly(hydroxyalkanoates) (mcl-PHAs) are bacterial thermoplastic elastomers with a large potential in medical applications. The present study provides a novel process to isolate and purify poly $([R]-$ 3-hydroxy- $\omega$-undecenoate-co-3-hydroxy- $\omega$-nonenoate-co-3-hydroxy- $\omega$-heptenoate) (PHUE) and poly([R]-3hydroxy- $\omega$-undecenoate-co-3-hydroxy- $\omega$-nonenoate-co-3-hydroxyoctanoate-co-3-hydroxy- $\omega$-heptenoate-co3-hydroxyhexanoate) (PHOUE) from Pseudomonas putida species. Three different types of activated charcoal were compared with regard to their capability to selectively remove impurities. The product 'Charcoal activated, powder, pure' from Merck was found to be most suitable. Using ethyl acetate as solvent, the polyesters were extracted from freeze-dried biomass at room temperature and simultaneously purified by addition of activated charcoal at the beginning of the extraction. The period of extraction was one hour and the ratio solvent to biomass was 15:1 (vol/wt). After extraction, the solids were separated by pressure filtration through a metallic lace tissue. The filtrate was again passed through the previously accumulated filter cake, followed by a second filtration through a $0.45 \mu \mathrm{m}$ membrane to remove finest coal particles. The resulting filtrate was concentrated, thus yielding polyesters whose quality and yield depended on the quantity of activated charcoal applied. For highly pure PHUE and PHOUE with low endotoxin levels, the optimum ratio of activated charcoal to solvent for extraction (V/V) was found to be 0.5 for PHUE and 0.25 for PHOUE. The yields with regard to the raw polymers amounted to 55 wt $\%$ for PHUE and 75 wt\% for PHOUE, which are acceptable for polymers that can be used for medical applications.
\end{abstract}

Keywords: Activated charcoal $\cdot$ Downstream processing $\cdot$ PHA $\cdot$ Polyhydroxyalkanoate $\cdot$ Purification

\section{Introduction}

Bacterial poly(hydroxyalkanoate)s (PHAs) whose monomers contain 6 to 14 carbon atoms are called medium chain length poly(hydroxyalkanoate)s (mcl-PHAs). Mcl-PHAs are thermoplastic elastomers. Length and functional groups of side chains can be controlled to some extent by feeding the cells with appropriate organic acids during biosynthesis in batch

${ }^{*}$ Correspondence: Dr. L. Thöny-Meyer

Empa, Swiss Federal Laboratories for Materials Science and Technology

Laboratory for Biomaterials

Lerchenfeldstrasse 5

$\mathrm{CH}-9014$ St. Gallen

Tel.: +4171274 7792

Fax: +41712747788

E-mail: linda.thoeny@empa.ch cultures, ${ }^{[1]}$ or more precisely under multiple-nutrient-limited growth conditions in chemostat cultures. ${ }^{[2,3]}$ When bearing carbon-carbon double bonds, mcl-PHAs can be used for chemical functionalization, ${ }^{[4,5]}$ e.g. cross-linking to obtain a biodegradable rubber. ${ }^{[6-8]}$ They also enable covalent binding of small molecules such as natural antifouling agents ${ }^{[9]}$ or the synthesis of organic/inorganic hybrid polymers. ${ }^{[10]}$ In order to separate PHA from other cell components such as cell walls, nucleic acids and peptides, appropriate extraction processes must be applied. Besides mechanical disruption, methods used most often are solvent extraction as well as chemical and enzymatic digestion. ${ }^{[11]}$ The recovery by using organic solvents prevails over aqueous treatment if PHA of high purity is required. PHA with a purity of about 90\% was obtained when the dry biomass from Pseudomonas putida was pre-treated with methanol for $5 \mathrm{~min}$ and subsequently extracted in a Soxhlet apparatus for $5 \mathrm{~h}$, followed by precipitation of PHA in cold methanol.[12] For the large-scale production of $\operatorname{poly}([R]-3$-hydroxyoctanoate- $\mathrm{co}$ 3-hydroxyhexanoate) (PHO), extraction or treatment of the biomass, respectively, using acetone, chloroform and sodium dodecyl sulfate was investigated. ${ }^{[13]}$ Acetone was found to give the highest yield. Purity of $99 \% \pm 0.2 \%$ was determined, when the
PHO concentrate had been precipitated in a volumetrically prepared mixture of $35 \%$ methanol, 35\% ethanol and 30\% water. When bacterial biopolymers are isolated for medical applications, contaminating pyrogenic compounds must be removed. Such compounds in the form of endotoxins (lipopolysaccharides) are present in the outer cell membrane of Gram-negative production strains. ${ }^{[14]}$ The United States Pharmacopeia (USP) approves maximum 20 endotoxin units (EU) per medical device, and 2.15 EU per device that contacts the cerebrospinal fluid. ${ }^{[15]}$ Low endotoxin PHO was achieved by extraction of the freezedried biomass with $n$-hexane at $50{ }^{\circ} \mathrm{C}$, followed by reducing the temperature of the extract to $0-5{ }^{\circ} \mathrm{C}$ after filtration. ${ }^{[16]}$ The precipitate thus obtained was subjected to temperature controlled re-dissolution and precipitation in 2-propanol, which resulted in a purity of over $99 \mathrm{wt} \%$. Yield and endotoxicity of mcl-PHA were investigated in dependence of the solvent used for extraction of the freeze-dried biomass. ${ }^{[17]}$ The results revealed that ethyl acetate and acetone have the same power of extraction as methylene chloride, but their use led to a polymer containing significantly less endotoxin. Endotoxin could be removed from aqueous solutions by filtration through activated charcoal. ${ }^{[18]}$ In the field of PHA, activated charcoal was also used 
for the purpose of purification, for example to decolorize and detoxify growth media prior to fermentation, ${ }^{[19]}$ to decolorize solutions of hydroxy acid methyl esters, ${ }^{[20]}$ or to purify poly(hydroxybutyrate) dissolved in chloroform. ${ }^{[21]}$

In a recent study, ${ }^{[22]}$ we developed a process to isolate $\mathrm{PHO}$ and poly $([R]-3$ hydroxy- $\omega$-undecenoate-co-3-hydroxy- $\omega$ nonenoate-co-3-hydroxy- $\omega$-heptenoate) (PHUE) from freeze-dried biomass of Pseudomonas putida species. Hereby, after solvent extraction, the crude extract was subjected to a filtration through activated charcoal. The resulting PHO and PHUE were of high purity and contained less than one endotoxin unit per g (EU/g). However, purification with activated charcoal caused a loss of material of $50 \%$ which consisted not only of impurities but also of PHO and PHUE, respectively. The present study aimed at optimizing the purification with activated charcoal, especially in terms of yield. For this purpose, bacterial PHUE and poly $([R]-3$-hydroxy- $\omega$-undecenoate-co-3-hydroxy- $\omega$-nonenoate- $c o-3$ hydroxyoctanoate-co-3-hydroxy- $\omega$-heptenoate-co-3-hydroxyhexanoate) (PHOUE) (Fig. 1) were extracted from freeze-dried biomass and purified with three different types of activated charcoal. The quality of the resulting polymers was evaluated as a function of the quantity of the activated charcoal. Critical parameters were yield, content of endotoxin and content of impurities characterized by UV/VIS spectrometry and size exclusion chromatography (SEC). The goal was to develop a downstream process which consists of only few simple laboratory steps, so that mcl-PHAs of high quality can be produced in a timesaving manner.

\section{Experimental Section}

\subsection{Materials}

PHUE was produced from $\omega$-undecenoate in a chemostat culture $\left(D=0.1 \mathrm{~h}^{-1}\right)$ of Pseudomonas putida GPo1 under dual (carbon, nitrogen) limited growth conditions $(\mathrm{C} / \mathrm{N}=12.5 \mathrm{~g} / \mathrm{g}) .{ }^{[3]}$ Gas chromatographic analysis revealed the following composition: $17.8 \mathrm{~mol} \%$ of 3-hydroxy- $\omega$ undecenoate, $63.2 \mathrm{~mol} \%$ of 3-hydroxy- $\omega$ nonenoate, and 19.0 mol\% of 3-hydroxy$\omega$-heptenoate. PHOUE was produced from octanoic and $\omega$-undecenoic acid in a chemostat culture $\left(D=0.1 \mathrm{~h}^{-1}\right)$ of Pseudomonas putida Gpo1 under dual (carbon, nitrogen) limited growth conditions $(\mathrm{C} / \mathrm{N}=12.5$ $\mathrm{g} / \mathrm{g}$ ). According to gas chromatographic analysis, the polymer consisted of 6.5 mol\% of 3-hydroxy- $\omega$-undecenoate, 22.9 mol\% of 3 -hydroxy- $\omega$-nonenoate, 58.2 mol\% of 3-hydroxyoctanoate, $5.3 \mathrm{~mol} \%$ of 3-hydroxy- $\omega$-heptenoate, and $7.1 \mathrm{~mol} \%$

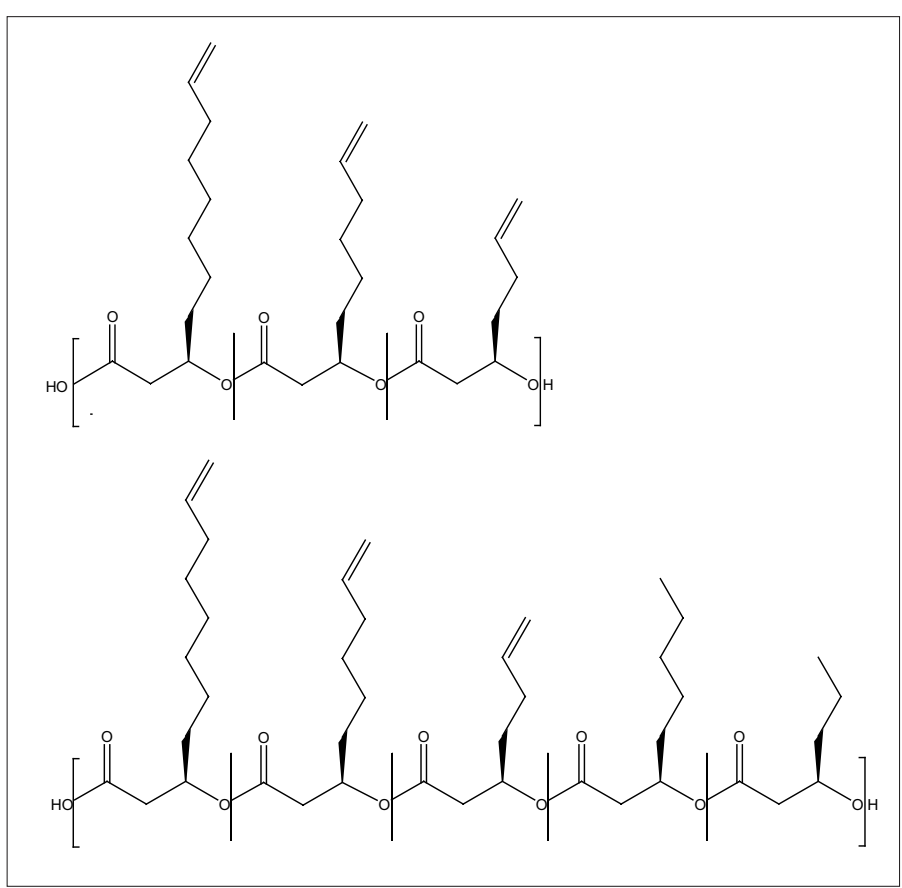

Fig. 1. Chemical structure of poly $([R]-3-h y d r o x y-\omega-$ undecenoate-co-3hydroxy- $\omega$-nonenoate-co-3-hydroxy- $\omega$-heptenoate) (PHUE) produced by Pseudomonas putida GPo1 when cultured on $\omega$-undecenoic acid (top), and of poly $([R]-3-h y d r o x y-\omega$-undecenoate-co-3-hydroxy- $\omega$ nonenoate-co-3-hydroxyoctanoate-co-3-hydroxy- $\omega$-heptenoate-co-3hydroxyhexanoate) (PHOUE) produced by Pseudomonas putida GPo1 when cultured on octanoic acid and $\omega$-undecenoic acid (bottom).

of 3-hydroxyhexanoate. Ethyl acetate was of $\mathrm{Ph}$. Eur. quality and was obtained from Hänseler AG, Herisau, Switzerland. Three types of activated charcoal were tested: i) charcoal activated, granular, about $1.5 \mathrm{~mm}$, pure, food grade, from Merck, Darmstadt, Germany, hereinafter called 'Merck granules', ii) charcoal activated, powder, pure, from Merck, Darmstadt, Germany, hereinafter called 'Merck powder', iii) Norit ${ }^{\circledR}$, from peat, steam activated, granular, 1-3 $\mathrm{mm}$ from Sigma Aldrich, Steinheim, Germany, hereinafter called Norit.

\subsection{Isolation of PHUE and PHOUE from Freeze-Dried Biomass}

A portion of $10 \mathrm{~g}$ of freeze-dried biomass was manually crushed to small pieces and mixed with $150 \mathrm{~mL}$ of ethyl acetate. The solids in the solvent were further reduced using a SilentCrusher M (Heidolph, Kelheim, Germany) equipped with a disperser $18 \mathrm{G} / \mathrm{M}$. Afterwards, y mL of activated charcoal were added, where y was 9 , $18,37.5,75$, or $112.5 \mathrm{~mL}$. The suspension was stirred for $1 \mathrm{~h}$ at room temperature. The phases were separated by pressure filtration (air, 2-5 bar) through a metallic lace tissue Duplex 15 (diameter of $47 \mathrm{~mm}$, G. Bopp, Zürich, Switzerland) using a 200 $\mathrm{mL}$ pressure vessel (SM 16249, Sartorius, Göttingen, Germany). In order to remove particles that swept along with the solution, the filtrate was again passed through the previously accumulated filter cake. The metallic lace tissue was replaced by a 0.45 $\mu \mathrm{m}$ membrane filter (RC 55, Whatman, Göttingen, Germany) and the solution was again filtered to remove finest coal particles. The filtrate was concentrated in a rotary evaporator at $40{ }^{\circ} \mathrm{C}$ and about 200 mbar. Residual solvent was removed by drying the gel-like solid in a vacuum dryer (VTR 5036, Heraeus, Hanau, Germany) for $24 \mathrm{~h}$ at $40{ }^{\circ} \mathrm{C}$ and $100 \mathrm{mbar}$. The pure polymer was weighed and afterwards stored at $-20^{\circ} \mathrm{C}$ to prevent autoxidation. ${ }^{[23]}$ The raw PHA was obtained in the same manner, but without addition of activated charcoal.

\subsection{Polymer Characterization}

Molecular weight fractionation was performed by size exclusion chromatography (SEC) using a differential refractive index detector (SEC apparatus: Viscotek, Houston, TX, USA). About $30 \mathrm{mg}$ of each polymer sample were dissolved in $10 \mathrm{~mL}$ of tetrahydrofuran (THF). Aliquots of $100 \mu \mathrm{L}$ of the polymer solution were injected and separated on three sequentially coupled SEC columns $(300 \mathrm{~mm} \times 8 \mathrm{~mm}$, pore sizes $10^{3}, 10^{5}$, and $10^{6} \AA$, PSS, Mainz, Germany) at $35^{\circ} \mathrm{C}$, applying a flow rate of $1 \mathrm{~mL} / \mathrm{min}$ with THF. Calibration was performed with 10 narrow standard polystyrene samples from PSS $\left(2 \times 10^{3} \mathrm{~g} / \mathrm{mol}\right.$ to $2.13 \times 10^{6} \mathrm{~g} / \mathrm{mol}$ ).

The partitions of 3-hydroxyalkanoates in PHUE and PHOUE were determined by 


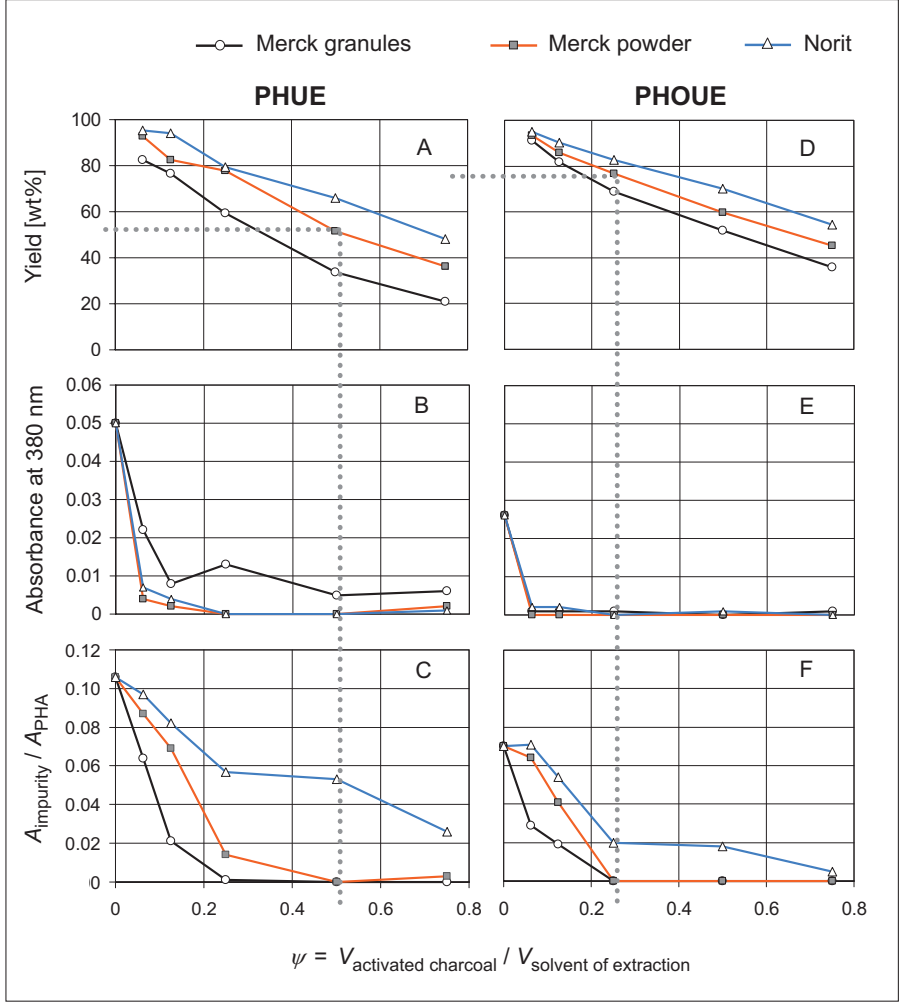

Fig. 2. Charts for evaluating quantity and type of activated charcoal for the purification of PHUE and PHOUE. The yield of PHA (A and D) is related to the corresponding raw polymer. The light absorption at 380 $\mathrm{nm}(\mathrm{B}$ and $\mathrm{E})$ is a measure for the unwanted yellow tinge of the polymer. $A_{\text {impurity }} / A_{\mathrm{PHA}}(\mathrm{C}$ and $\mathrm{F})$ means the ratio of the area of the impurity peak to the area of the adjoining PHA peak in chromatograms obtained by SEC. The values of the raw polymers can be seen at the volume ratio $\psi=0$. The dotted lines show the optimum choice of an activated charcoal for PHUE ( $A$ to $C$ ) and PHOUE (D to $F$ ) when highest quality is required.

gas chromatography (GC) after conversion of the polymer into the methyl ester of the monomers using boron trifluoride in methanol. ${ }^{[24]}$ The analyses of the resulting hydroxyalkenoic and hydroxyalkanoic acid methyl esters were performed with a gas chromatograph (GC Trace 2000 Series, CE Instruments, Rodano, Italy) equipped with a flame ionization detector and a capillary column DB-Wax $(30 \mathrm{~m} \times 0.32 \mathrm{~mm}$, film thickness: $0.3 \mu \mathrm{m}$, Agilent, Santa Clara, CA, USA).

UV/VIS absorption spectra were taken from solutions of PHUE and PHOUE in chloroform at ambient temperature using a Synergy Mx UV/VIS spectrophotometer from Biotek Instruments $\mathrm{GmbH}$, Luzern, Switzerland. Endotoxicity was determined by means of the Limulus Amebocyte Lysate (LAL) assay Endosafe-PTS (Charles River, L'Arbresle Cedex, France). Samples were prepared by dissolving $200 \mathrm{mg}$ of polymer in $13 \mathrm{~mL}$ of chloroform and casting into depyrogenized glass Petri dishes $(\mathrm{d}=4 \mathrm{~cm})$, followed by drying at $40{ }^{\circ} \mathrm{C}$ and 100 mbar for $24 \mathrm{~h}$. In order to extract endotoxins, samples were incubated with $3 \mathrm{~mL}$ of endotoxin-free water (LAL Reagent Water Endosafe) at $37{ }^{\circ} \mathrm{C}$ for $24 \mathrm{~h}$.
Aliquots of $200 \mu \mathrm{L}$ were withdrawn and each mixed with $200 \mu \mathrm{L}$ of dispersing agent BD100 (Endosafe). Samples which contained more than $10 \mathrm{EU} / \mathrm{g}$ of endotoxin were diluted 20 to 50 times with dispersing agent BD 100. The resulting solutions were measured according to the manufacturer's instructions with the spectrophotometer Endosafe-PTS using cartridges PTS2001. PTS cartridges allow testing of sample and positive sample control in duplicates. When the endotoxin content of the raw polymers was determined, water extracts were diluted 10 times with endotoxin-free water and no dispersing agent was added. In this case, measurements were taken with the cartridge Endosafe PTS2005.

\section{Results}

\subsection{Comparison of Three Types of Activated Charcoal}

The PHAs were extracted from freezedried biomass with ethyl acetate and simultaneously purified by addition of different quantities of activated charcoal to the mixture of extraction. Afterwards, the mixture was filtered in three steps and the filtrate was concentrated, thus yielding purified polymers. Three types of activated charcoal were compared: Merck granules, Merck powder, and Norit. Their suitability to purify PHUE and PHOUE was evaluated on the basis of their capability to remove impurities and of the resulting yield related to the raw polymers. In Fig. 2, the results for PHUE (A to C) and PHOUE (D to F) are depicted in function of the ratio $\psi$ of activated charcoal to solvent used for extraction (V/V). The values of the raw polymers can be seen at $\psi=$ 0 . With increasing $\psi$, the yield decreased almost linearly to 20-50 wt\% (PHUE) or to $35-55 \mathrm{wt} \%$ (PHOUE), depending on the type of activated charcoal used (Charts A and D). Charts B and E show the light absorbance of the raw and pure PHA at 380 nm. Since molar extinction coefficients differ over log scales, direct conclusions about the quantity of impurities cannot be drawn from UV/VIS absorbance without knowing the substances responsible for $a b-$ sorption. Nevertheless, the UV/VIS absorbance was judged to be critical, because it gradually decreased from $380 \mathrm{~nm}$ to longer wave length and came to zero in the middle part of the visible spectrum (see spectra a and $b$ in Fig. 3). Such absorption spectra go along with a yellow tinge of the polymer, which is not acceptable for products of high quality. According to the results of the UV/VIS measurements (Fig. 2 and 3 ), compounds causing this tinge were selectively removed by all types of activated charcoals except for Merck granules in case of PHUE (Fig. 2B). In Charts $\mathrm{C}$ and $\mathrm{F}$, the ratio $A_{\text {impurity }} / A_{\mathrm{PHA}}$ is shown in function of $\psi$. It refers to the areas of the SEC peaks at $30-30.5 \mathrm{~mL}$ (impurity) and at 24-26 mL (PHA) (see Fig. 4), respectively, and is a measure for the removal of polymeric impurities being contained in PHUE and PHOUE. Their complete removal required a $\psi$ of 0.5 for PHUE and of 0.25 for PHOUE (Fig. 2C and F). The selectivity of Norit for polymeric impurities was found to be low. These impurities were not even removed completely at $\psi=0.5$, neither in case of PHUE nor of PHOUE.

\subsection{Molecular Weight Fractionation}

Fig. 4A shows the SE chromatograms of raw PHUE and PHOUE which have not been treated with activated charcoal. The weight average molecular weight $M_{\mathrm{w}}$ of PHUE was found to be $135 \mathrm{kDa}$ accompanied by a polydispersity (PDI) of 3.3. For PHOUE, an $M_{\mathrm{w}}$ value of $201 \mathrm{kDa}$ and a PDI of 3.6 were determined. As can be seen from Fig. 4A, both raw polymers contained polymeric impurities, indicated by a peak at $30.4 \mathrm{~mL}$ and corresponding to an $M_{\text {w }}$ of about $9 \mathrm{kDa}$. The peaks overlap in both cases. It could be observed by SEC that not only impurities but also PHUE and 


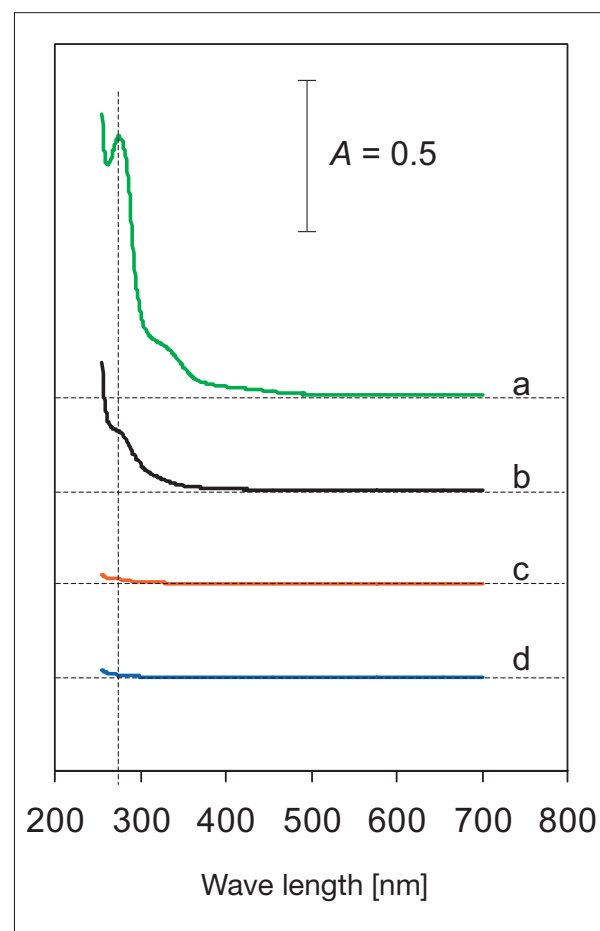

Fig. 3. UV/VIS spectra of raw PHUE (a) and PHUE purified with Merck granules (b), Merck powder (c), and Norit (d). Purification was performed applying the ratio $\psi=0.25=$ volume of activated charcoal / volume of solvent for extraction. Spectra were taken from polymers dissolved in chloroform and diluted to identical concentration. $A=$ absorbance.

PHOUE were retained by activated charcoal. Fig. 4B shows an example in which PHUE was treated with different quantities of Merck powder during extraction. The common intersection point of three curves at $26.5 \mathrm{~mL}$ (raw PHUE, $\psi=0.06$ and 0.12 ) can be explained by adsorption of impurities causing a decrease of the peak at $30.5 \mathrm{~mL}$ and a corresponding increase of the main peak due to the standardization to identical peak areas. Once the impurities had been removed to some extent, low molecular PHUE was additionally retained and the main peak consequently shifted towards lower retention volumes $(\psi=0.25$ and 0.75 , see arrows). The selective removal of low molecular PHUE caused a decrease of the polydispersity from $2.1(\psi=0.25)$ to $1.6(\psi=0.75)$, indicating a decrease of the broadness of the molecular weight distribution. When $\psi$ was increased from 0 to 0.75 , the $M_{\mathrm{w}}$ of PHUE continuously increased from 135 to $219 \mathrm{kDa}$ while the PDI decreased from 3.3 to 1.6. Analogous effects were observed in case of the other charcoals and with PHOUE. However, the change of $M_{\mathrm{w}}$ and PDI caused by Norit was less pronounced, because this activated charcoal showed i) low selectivity in terms of impurities, especially for the ones of PHUE, and ii) rather low adsorption capacity for the material in question.

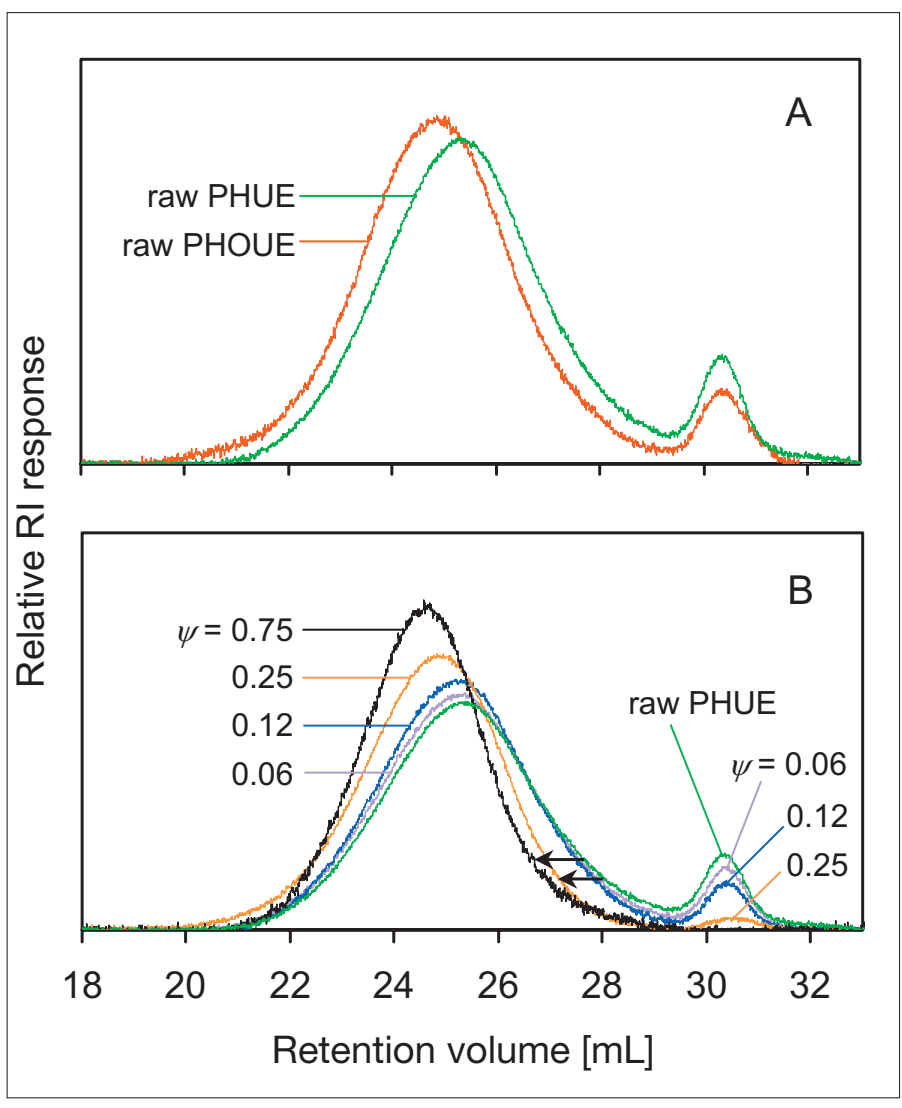

Fig. 4. Size exclusion chromatograms of raw PHUE and raw PHOUE which were extracted from freeze-dried biomass without addition of activated charcoal (A), and size exclusion chromatograms of PHUE extracted from freeze-dried biomass in the presence of different quantities of activated charcoal (B). The quantities of activated charcoal are given as a ratio $\psi=$ volume of activated charcoal / volume of solvent used for extraction. All chromatograms are normalized to identical peak areas. The arrows point to the adsorption of low molecular PHUE, which occurred as soon as the impurities had almost been removed.

\subsection{Endotoxicity}

Both raw polymers were contaminated with more than $50 \mathrm{EU} / \mathrm{g}$ of endotoxin. Since medical devices such as vascular grafts, heart valves or catheters often weigh only few grams, and the limit approved by USP is $20 \mathrm{EU} /$ device, ${ }^{[15]}$ the basic materials require endotoxin contents of less than 1 to $2 \mathrm{EU} / \mathrm{g}$. The capability of the three types of activated charcoal to adsorb endotoxins was examined by application of $\psi=0.25$. After purification with Merck granules or Merck powder, the endotoxin content of PHUE was still $22 \mathrm{EU} / \mathrm{g}$ or $13 \mathrm{EU} / \mathrm{g}$, respectively. When PHUE was purified with Norit, the amount of endotoxin could not be determined accurately, because the spike recovery was too low. Norit granules were accompanied by very fine powder. Some small particles probably passed through the filter and contaminated the polymer, where they could interfere with the measurement by partial adsorption of the spike. Removal of endotoxins from PHOUE was more successful: from PHOUE treated with Merck granules or Merck powder, endotoxins were almost completely eliminated; the contents were found to be 0.4 or $0.7 \mathrm{EU} / \mathrm{g}$, respectively. A content of $3.1 \mathrm{EU} / \mathrm{g}$ was measured after purification using Norit. When applying Merck granules at $\psi=0.5$ in a separate filtration step, ${ }^{[22]}$ we obtained $\mathrm{PHO}$ and PHUE with an endotoxin level of less than $1 \mathrm{EU} / \mathrm{g}$. Similar values were obtained in another project, where PHOUE was produced with the following molar ratios of octanoic to $\omega$-undecenoic acid: 90/10, $75 / 25$, and 50/50. Endotoxin contents of all samples decreased from 20 to $50 \mathrm{EU} / \mathrm{g}$ to values below $1 \mathrm{EU} / \mathrm{g}$ by purification with Merck granules (data not shown).

\section{Discussion}

We recently developed a downstream process which uses Merck granules to purify PHO and PHUE. ${ }^{[22]}$ The process consisted of nine steps including a separate filtration of the PHA solution through a column filled with activated charcoal as well as subsequent precipitation of $\mathrm{PHO}$ and PHUE to remove remaining impuri- 
ties. The filtration step lasted $5 \mathrm{~h}$ and had to be repeated if the filtrate was still yellowish. In the new process reported here, the two steps mentioned above have been eliminated. Purification with activated charcoal is now performed simultaneously with the extraction of PHA from freezedried biomass. After filtration, the PHAs are isolated by direct concentration of the filtrate without prior precipitation. The novel process allows the production of PHUE and PHOUE of high quality.

As can be learned from Fig. 2, Merck powder applied with $\psi=0.5$ led to PHUE free from detectable polymeric impurities $\left(A_{\text {impurity }} / A_{\mathrm{PHA}}=0\right)$ and yellow tinge $\left(A_{380}=\right.$ 0 ) (dotted line on the left). The resulting yield of $55 \mathrm{wt} \%$ might be acceptable for a polymer that can be used for medical purposes. A similar quality was achieved by analogous application of Merck granules, but the yield decreased to $35 \mathrm{wt} \%$. PHUE of middle quality but in high yields was obtained by treatment with Merck powder at $\psi=0.25$. The resulting polymer was colorless and contained only small amounts of polymeric impurities. In contrast, application of Merck granules at $\psi=0.25$ led to $60 \mathrm{wt} \%$ of a yellowish PHUE free of polymeric impurities. In order to achieve low endotoxin levels, the ratio of activated charcoal had to be increased to $\psi=0.5$ at the expense of yield. However, application of higher quantities of Merck powder $(\psi>$ 0.12 ) caused unwanted loss of low molecular PHUE (Fig. 4B, arrows). Similar effects were observed in case of the two other activated charcoals and with PHOUE (data not shown). With Norit, PHUE could not be purified efficiently; the highest $\psi$ value was still too low for complete removal of polymeric impurities (Fig. 2C). In case of PHOUE, application of Merck powder or Merck granules with a volume ratio $\psi=$ 0.25 yielded a pure polymer (dotted line on the right) with an endotoxin content of less than $1 \mathrm{EU} / \mathrm{g}$. The yield with respect to the raw polymer was found to be 75 or $70 \mathrm{wt} \%$, respectively, which is acceptable for a product that can be used for medical applications. If high yield at the expense of the quality was required, Merck granules could be applied with a ratio of 0.12 or even of 0.06. In order to remove the polymeric impurities from PHOUE with Norit, a high volume ratio $\psi$ of 0.75 was necessary, which entailed a yield of only $55 \mathrm{w} \%$. In comparison to the downstream process recently developed, ${ }^{[22]}$ the required quantity of activated charcoal to produce highly pure PHA could be halved in the case of PHOUE. On the basis of the investigated parameters, the following rating for the suitability to purify PHUE or PHOUE was found: Merck powder $>$ Merck granules $>>$ Norit. A disadvantage of these activated charcoals is their low adsorption capacity for organic acids and their salts. When the biomass of Pseudomonas putida GPo1 contained $\omega$-undecenoate, for example, PHUE purified by activated charcoal was found to be contaminated by this educt. Therefore, it is of importance that all carbon substrates are metabolized to completion during biosynthesis of PHA. This can be achieved by continuous cultivation when the carbon to nitrogen ratio in the medium feed are controlled accurately. ${ }^{[25]}$ Interestingly, PHUE required significantly more activated charcoal for the purification than PHOUE. One can argue that this fact is based on the higher content of impurities found in PHUE (see Fig. 2 at $\psi=0$ and Fig. 4A). However, with increasing $\psi$, the decrease of the yield continued without changing the slope after complete adsorption of the impurities (Fig. 2A and D). This suggests that PHAs were strongly adsorbed by activated charcoals and hence were in competition with impurities for adsorption sites. Due to the pore size distribution of the activated charcoals used, low molecular PHAs were adsorbed preferentially (see Fig. 4B). Since PHUE has a lower $M_{\mathrm{r}}$ than PHOUE (Fig. 4A), more PHUE molecules could enter into the pores and hence the adsorption capacity for impurities became lower. This may be the explanation why different quantities of activated charcoal were needed to purify PHUE and PHOUE.

\section{Conclusions}

The present study provides an optimized downstream process that permits the isolation and purification of PHUE and PHOUE from freeze-dried biomass using activated charcoal. Three charcoals were compared in terms of yield of PHA and removal of impurities. Merck powder excelled at its specificity for impurities, whereas Norit was not suitable for the intended purpose. Extraction from biomass and purification from soluble impurities were performed simultaneously by addition of activated charcoal just at the beginning of the extraction process. Subsequent separation of the solids by filtration followed by concentration of the filtrate led to a pure polymer that is almost free of endotoxins and thus is suitable for medical applications.

In the present process, all steps involving separate filtration through activated charcoal or precipitation of PHAs to remove impurities could be eliminated. Thus, time, energy, solvent and non-solvent can be saved. The developed process is better suited to isolate and purify PHUE and PHOUE. However, the method needs further optimization to improve the yield of PHUE. Possible approaches include i) fine-tuning of the volume ratio $\psi$, ii) op- timization of the period of extraction, and iii) the inclusion of further types of activated charcoal or of zeolite.

\section{Acknowledgement}

We thank Roland Hany and Daniel Rentsch for verifying our gas chromatographic results by ${ }^{1} \mathrm{H}-$ and ${ }^{13} \mathrm{C}-\mathrm{NMR}$.

Received: September 6, 2010

[1] Y. B. Kim, R. W. Lenz, R. C. Fuller, J. Polymer Sci. Polymer Chem. 1995, 33, 1367.

[2] R. Hartmann, R. Hany, T. Geiger, T. Egli, B. Witholt, M. Zinn, Macromolecules 2004, 37, 6780.

[3] R. Hartmann, R. Hany, E. Pletscher, A. Ritter, B. Witholt, M. Zinn, Biotechnol. Bioeng. 2006, 93, 737.

[4] B. Hazer, A. Steinbüchel, Appl. Microbiol. Biotechnol. 2007, 74, 1

[5] E. Renard, V. Langlois, P. Guerin, Corros. Eng. Sci. Technol. 2007, 42, 300.

[6] K. D. Gagnon, R. W. Lenz, R. J. Farris, R. C. Fuller, Polymer 1994, 35, 4358.

[7] K. D. Gagnon, R. W. Lenz, R. J. Farris, R. C. Fuller, Polymer 1994, 35, 4368.

[8] G. J. M. de Koning, H. H. M. van Bilsen, P. J. Lemstra, W. Hazenberg, B. Witholt, H. Preusting, J. G. van der Galiën, A. Schirmer, D. Jendrossek, Polymer 1994, 35, 2090.

[9] R. Hany, C. Böhlen, T. Geiger, M. Schmid, M. Zinn, Biomacromolecules 2004, 5, 1452.

[10] R. Hany, R. Hartmann, C. Böhlen, S. Brandenberger, J. Kawada, C. Löwe, M. Zinn, B. Witholt, R. H. Marchessault, Polymer 2005, 46, 5025 .

[11] N. Jacquel, C.-W. Lo, Y.-H. Wei, H.-S. Wu, S. S Wang, Biochem. Eng. J. 2008, 39, 15.

[12] X. Jiang, J. A. Ramsay, B. A. Ramsay, J. Microbiol. Meth. 2006, 67, 212.

[13] Y. Elbahloul, A. Steinbüchel, Appl. Environ. Microbiol. 2009, 75, 643.

[14] D. Petsch, F. B. Anspach, J. Biotechnol. 2000, 76, 97.

[15] 'United States Pharmacopeia and National Formulary', (USP29-NF24) United States Pharmacopeial Convention, Rockville, 2005, Chapter 161.

[16] P. Furrer, S. Panke, M. Zinn, J. Microbiol. Meth. 2007, 69, 206.

[17] P. Furrer, M. Schmid, A. Hinz, E. Pletscher, S. Panke, M. Zinn, Eur. Cells Mat. 2004, 7 (Suppl. 2), 30 .

[18] A. S. Pegues, S. S. Sofer, R. E. McCallum, L. B. Hinshaw, Int. J. Artif. Organs 1979, 2, 153.

[19] T. M. Keenan, J. P. Nakas, S. W. Tanenbaum, J. Ind. Microbiol. Biotechnol. 2006, 33, 616.

[20] W. He, W. Tian, G. Zhang, G.-Q. Chen, Z Zhang, FEMS Microbiol. Lett. 1998, 169, 45.

[21] R. N. Reusch, H. L. Sadoff, Proc. Natl. Acad. Sci. U.S.A. 1988, 85, 4176.

[22] B. Wampfler, T. Ramsauer, S. Rezzonico, R. Hischier, R. Köhling, L. Thöny-Meyer, M. Zinn, Biomacromolecules 2010, 11, 2716.

[23] M. Schmid, A. Ritter, A. Grubelnik, M. Zinn, Biomacromolecules 2007, 8, 579.

[24] P. Furrer, R. Hany, D. Rentsch, A. Grubelnik, K. Ruth, S. Panke, M. Zinn, J. Chromatogr., A 2007, 1143, 199.

[25] M. Zinn, B. Witholt, T. Egli, J. Biotechnol. 2004, 113, 263 